\title{
The effects of position cues on the appearance of stimulus elements in a bistable apparent movement display
}

\author{
J. TIMOTHY PETERSIK and AMY ROSNER \\ Ripon College, Ripon, Wisconsin
}

\begin{abstract}
A modified version of the Ternus display was used to assess the relative effects of element position cues on reports of group and end-to-end movement. In this display, two rows of stimulus elements are joined by connecting lines. In one version of the display, the connecting lines remain stationary across frames, facilitating the interpretation that the associated stimulus elements also remain stationary. In another version of the display, one end of the connecting lines shifts horizontally from frame to fame, facilitating the interpretation that the associated stimulus elements have also shifted. The experiment showed that when the connecting lines remain stationary, reports of end-to-end movement increase, regardless of the interstimulus interval (ISI) at which the frames alternate. When the connecting lines shift, reports of group movement increase, regardless of ISI. Theoretical interpretations of the results involving both relatively lowlevel motion signals and higher order perceptual influences are considered.
\end{abstract}

Apparent movement (AM) is an illusory percept of motion that can occur when at least two spatially separated stimulus elements are presented at different moments in time. Many studies of AM have shown that the nature of the percept reported can be influenced by a variety of cues or contexts. For example, Benussi (1913; cited in Boring, 1942) demonstrated that an apparent obstruction between two alternately presented stimuli would result in a path of AM around the obstacle. More recently, Anstis and Ramachandran (1986) have shown that stimulus elements whose shortest potential path of AM would be horizontal can be seen as moving through a longer Vshaped path when collections of background elements are simultaneously moving through such a path. Green and Odom (1986) found that stimulus matches in AM can be determined by the 3-D proximity of the elements as produced with stereoscopic viewing. Sigman and Rock (1974) showed that percepts of either AM or stationarity involving the same elements can be obtained, depending on whether the entire stimulus field promotes an interpretation favoring motion or not. Of greatest relevance to the present study, Shepard and Zare (1983) demonstrated an effect called "path-guided apparent movement." In this phenomenon, two alternately presented stimulus elements can be seen to follow a curved path of motion when a path cue is made available; in the absence of the path cue, AM is seen through a shorter straight-line path. Whereas Shepard and Zare (1983) provided a path cue that guided $\mathrm{AM}$, in the present experiment we provided

Correspondence may be addressed to J. Timothy Petersik, Department of Psychology, Ripon College, P.O. Box 248, Ripon, WI 54971. position cues (i.e., cues that suggested either a particular position change or a lack thereof) in a normally bistable AM display and found that either of two percepts can be favored by selection of the appropriate position cues.

A recently completed review of the literature regarding short-range (SR) and long-range (LR) processes in AM (Petersik, 1989) revealed that virtually all of the strongest demonstrations of cue effects involved the LR process. ${ }^{1}$ In the present study, we sought to show that position cues could affect percepts that are mediated by both the LR and the SR processes. Thus, we used variations of what has been called the Ternus display (Ternus, 1938). In a simple version of this display, a frame containing three dots evenly spaced in a row alternates with a second frame containing the same three dots shifted horizontally in one direction by the center-to-center distance separating them (see Figure 1). Previous work (e.g., Pantle \& Petersik, 1980; Pantle \& Picciano, 1976; Petersik \& Pantle, 1979) has shown that when the conditions of presentation favor the SR process (e.g., short, dark interstimulus intervals, ISIs) observers report end-to-end motion involving only the unpaired outer dots. However, when conditions of presentation favor the LR process (e.g., long or illuminated ISIs), observers report back and forth movement of the entire collection of dots, known as "group movement." Since the frames containing the stimulus elements do not themselves change, while only the conditions of presentation do, it is clear that the two percepts represent alternate solutions to the "correspondence problem" (Ullman, 1979). There is considerable evidence that the end-to-end movement percept is in fact mediated by the SR process, whereas the group-movement percept is mediated by the LR process (see Petersik, 1989, for a review). 

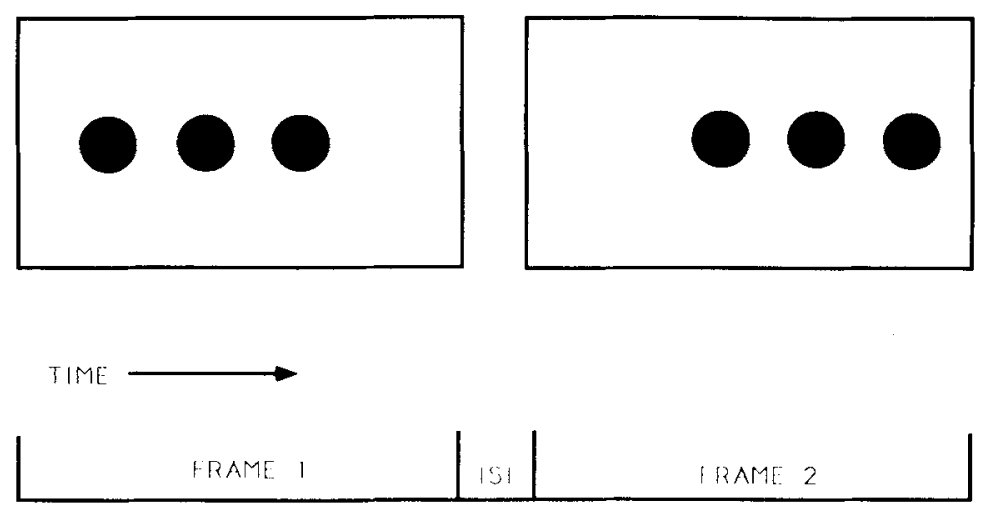

Figure 1. An example of the typical Ternus display used to study short- and longrange motion percepts (e.g., see Pantle \& Picciano, 1976). The rightmost dots in the first frame overlap the leftmost dots in the second frame. This figure is illustrative only; the distances shown here are not scaled to those used in the experiment.

In order to demonstrate the influence of position cues on percepts of group and end-to-end movement, we first added a second, lower, row of stimulus dots to each frame of the Ternus display (see Figure 2). Stimulus elements of the lower row were either not displaced from frame to frame (Figure 2, panels a, c,e) or displaced as in the usual Ternus display (Figure 2, panels b, $d, f$ ). Those in the upper, target, row of elements were always displaced horizontally by one unit of distance separating the dots. Next, we drew connecting lines between pairs of dots in the upper and lower rows in order to establish a position cue: The lines always originated from the same two dots in the lower row; however, their termination in the upper row varied between the stimulus dot immediately above the origin and the next dot to the right (see Figure 2). If the AM percepts produced by the Ternus display are determined solely by factors related to the nature of the presentation of the stimulus frames (e.g., by stimulus duration and ISI), then the existence of position cues should not affect the percentage of reports of group and end-to-end movement, relative to control conditions (Figure 2, panels a and b). However, if position cues influence the AM percept, it would be expected that displays in which the connecting lines remain vertical from frame to frame would increase the number of reports of end-to-end movement. Displays in which the tops, or terminals, of the connecting lines are shifted horizontally from frame to frame would be expected to produce more reports of group movement.

\section{METHOD}

\section{Subjects}

The subjects consisted of 20 volunteers from Ripon College psychology classes. All subjects reported normal or corrected-to-normal vision.

\section{Apparatus and Stimuli}

A three-channel tachistoscope (Scientific Prototype, Model GB) was used to present the stimulus frames. Subjects sat on a stool inside a black booth and looked through a set of eyeholes to view the screen. Other than that provided by the stimuli, no light was visible inside the viewing booth. Viewing distance was $153 \mathrm{~cm}$, and at this distance the stimulus cards subtended $6.7^{\circ}$ horizontally and $4.86^{\circ}$ vertically.

Each stimulus consisted of two computer-generated pictures that contained both an upper and a lower row of three black stimulus dots on a white background (see Figure 2). The diameter of each dot subtended $.49^{\circ}$, and the center-to-center separation between adjacent dots subtended $1.05^{\circ}$. Luminance of the white background was $4.5 \mathrm{~cd} / \mathrm{m}^{2}$, and luminance of the stimulus dots was $0.8 \mathrm{~cd} / \mathrm{m}^{2}$, resulting in a Michelson contrast of $70 \%$. The center of the upper row of dots was $0.75^{\circ}$ above the center of the screen, whereas the lower row was $0.75^{\circ}$ below. For all stimuli, the dots in the upper row of the second frame were shifted to the right by $1.05^{\circ}$ (the center-to-center distance separating the dots) relative to the dots in the first frame. For half of the stimuli, the dots in the bottom row of the second frame were similarly shifted; for half, they remained in their original positions. This manipulation was used to determine the extent to which the actual motion or stationarity of the dots in the bottom row might influence reports of end-to-end and group movement of the dots in the upper row.

One third of the stimulus frames contained no connecting bars (control condition). For the control condition in which the lower dots remained stationary, únbiased estimates of end-to-end and group movement as a function of ISI could be obtained. For the control condition in which the lower dots were shifted from frame to frame, an estimate of the influence of the motion of the lower dots, if any, could be obtained (see Figures $2 a$ and $2 b$ ). One third of the stimulus frames contained connecting bars that remained stationary across alternating frames (see Figures $2 c$ and $2 d$ ). Finally, one third of all stimulus frames contained connecting bars whose upper terminations shifted from left to right from the first to second AM frame (see Figures $2 \mathrm{~d}$ and $2 \mathrm{e}$ ).

\section{Procedure}

The subjects were run individually in single sessions lasting approximately $45 \mathrm{~min}$. Each subject was instructed to look toward the center of the screen during the motion displays and to base judgments on the motion of the upper row of dots. A trial consisted of four cycles of the stimulus frames, where one cycle was defined as Stimulus ${ }_{1}$-ISI-Stimulus - -ISI. ISIs were not illuminated. Following each trial, the subject's task was to inform the experimenter whether the upper dots appeared to shift back and forth as a group, 


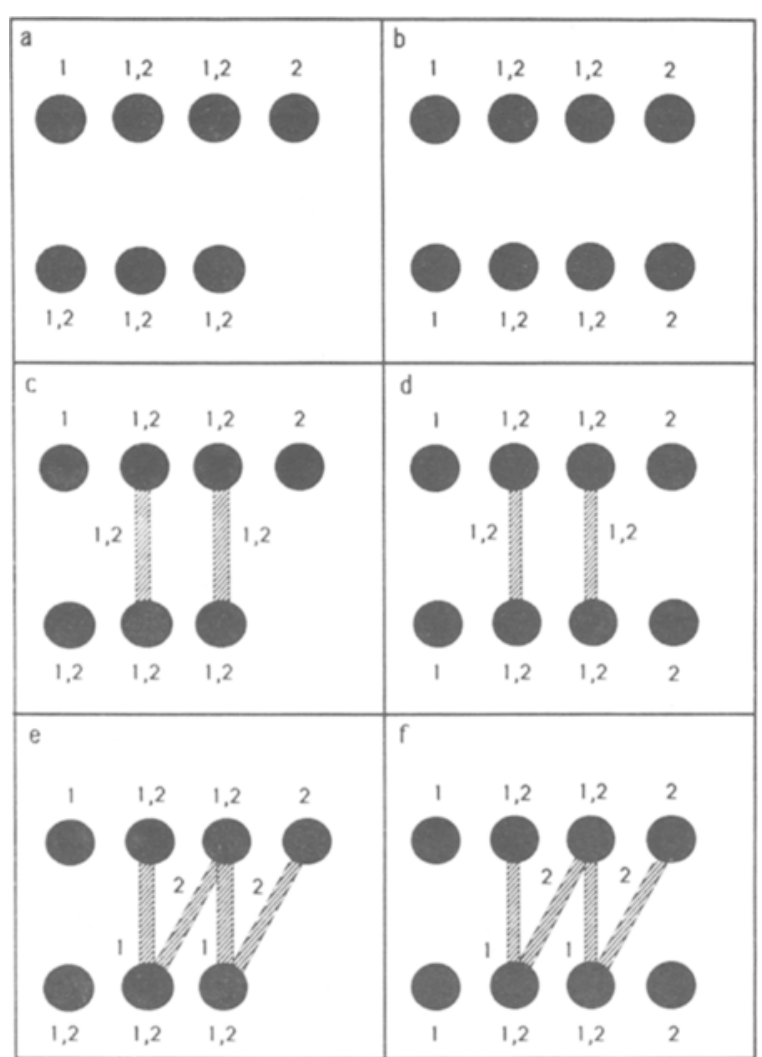

Figure 2. Modifications of the Ternus display used in the present experiment. In each frame, elements labeled "1" appeared in the first AM frame; those labeled "2" appeared in the second. Panels $a, c$, and e show displays in which the lower row of dots remained stationary. Panels b, $d$, and $f$ show displays in which the lower row of dots shifted from frame to frame. Panels a and b show control conditions; $c$ and $d$, connecting lines that remained vertical from frame to frame; $e$ and $f$, connecting lines whose top termini shifted from frame to frame.

or whether two appeared to remain stationary with one dot moving from end to end. No other percepts were ever reported.

The subjects were run in five randomized blocks of trials. Each block consisted of 12 trials resulting from the six stimulus types shown in Figure 2, paired with two ISIs (10 and $80 \mathrm{msec}$ ). The subjects' scores were the percentages of group-movement responses for the 12 stimuli across the five blocks.

\section{RESULTS}

The two panels of Figure 3 show, respectively, the percentages of group-movement reports for the stimuli in which the lower row of dots was not shifted or shifted. The data were analyzed by a $2 \times 2 \times 3$ (ISI $\times$ lower dot shift $\times$ cue type) repeated measures analysis of variance. As Figure 3 suggests, there was a significant main effect of ISI $[F(1,19)=49.13, p<.001]$; for each type of stimulus, more group-movement responses were produced with the 80 -msec ISI than with the 10 -msec ISI. Additionally, there was a significant main effect of context type $[F(2,38)=62.76, p<.001]$. This was exem- plified by the fact that the stimulus cues designed to produce LR (group) movement and SR (end-to-end) movement resulted in the most and fewest reports of group movement, respectively, regardless of ISI. There was no significant influence of the organization of the dots in the lower row of these displays $[F(1,19)=.50, p>.05]$, nor was there any significant interaction involving this factor, suggesting that the lower dots did not contribute to the position-cue effects reported here.

The data did reveal a significant interaction between ISI and cue type $[F(2,38)=11.04, p<.001]$. Since all simple main effects involving these factors proved to be significant, the interaction can be interpreted as reflecting the shallower slope obtained with the stimuli cued to produce LR (group) movement. This, in turn, is most likely due to a ceiling effect: Since the stimuli designed to produce LR movement yielded group-movement reports $70 \%-80 \%$ of the time with the $10-\mathrm{msec}$ ISI, the possible increase obtainable with the $80-\mathrm{msec}$ ISI was limited to no more than $20 \%-30 \%$.

\section{DISCUSSION}

The results can be considered from the standpoint of how the position cues influenced the reports of end-toend and group movement at each ISI. With the control stimuli, the 10 -msec ISI produced end-to-end movement on over $60 \%$ of the trials. When the position cues facilitated the stationarity interpretation of the "overlapping" dots in these displays, the end-to-end movement percept increased by $32.5 \%$ on the average. On the other hand, stimuli whose cues suggested position change decreased the reports of end-to-end movement by $38.5 \%$ on the average.

Results obtained with the 80 -msec ISI are not surprising, given previous reports that LR motion percepts are susceptible to context or cue effects (see Petersik, 1989). In the present experiment, the control stimuli yielded group-movement reports on $88.5 \%$ of all trials. Stimuli whose cues facilitated position change could not have much opportunity to raise that percentage, and in fact the increase was only $7.5 \%$. However, stimuli designed to facilitate stationarity decreased reports of group movement by $52 \%$ on the average.

Given that the data demonstrate effects of both types of position cues on both types of motion percepts (with a visible, although minimal, effect of the position-change cue on the group-movement percept), the theoretical challenge focuses on how to account for such effects. We start with the assumption that in the case of the control stimuli, the group-movement percept is mediated by the LR process while at least the stationary appearance of the overlapping elements in end-to-end movement is mediated by the SR process (Braddick \& Adlard, 1978; Pantle \& Petersik, 1980; Petersik, 1989). ${ }^{2}$ Thus, one possible explanation of the present results is that signals generated by the connecting bars themselves interacted with the Ternus elements to facilitate either LR or SR mo- 
(a)

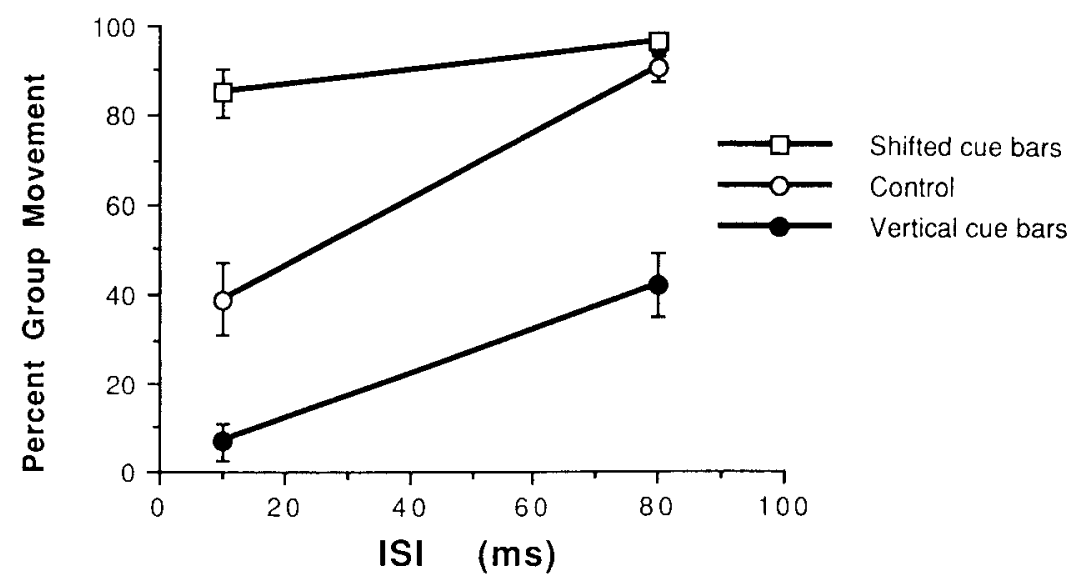

(b)

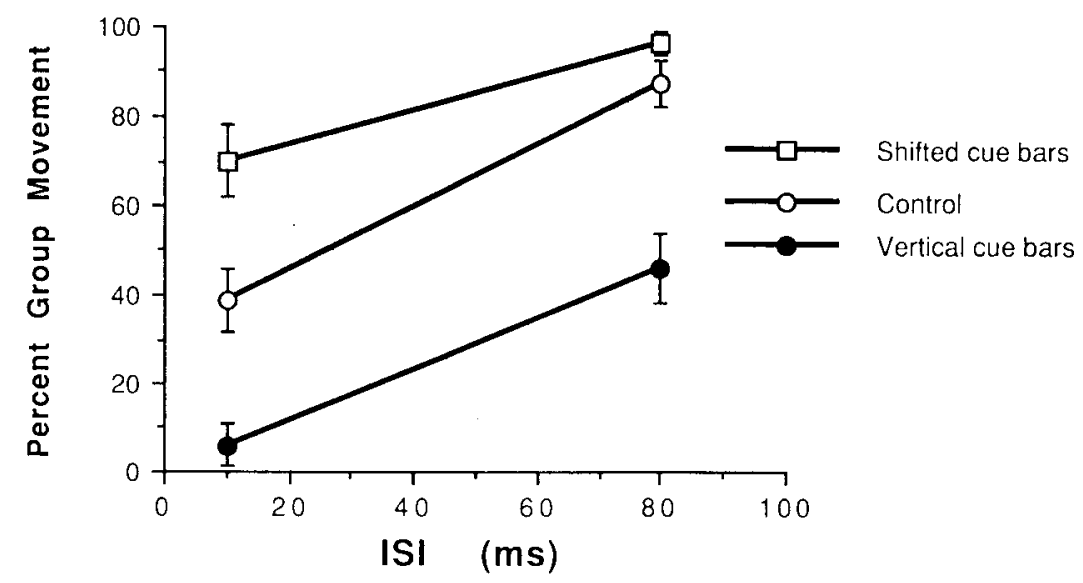

Figure 3. Results of the experiment, with percentage of group movement responses shown as a function of interstimulus interval for each condition of the experiment. Panel a shows results obtained with displays in which the lower dots remained stationary; panel b, displays in which the lower dots shifted from frame to frame. Error bars reflect $\pm 1 S E M$.

tion signals. There already is considerable evidence that LR motion can be influenced by stimulus contexts or cues (see Petersik, 1989). In addition, the SR process is known to exhibit global cooperativity, both with random-dot displays (Change \& Julesz, 1984) and with larger contourcontaining stimuli (Petersik, 1990). Therefore, there is reason to believe that the bars themselves generated LRmotion or SR-stationarity signals that added to signals generated by the dots themselves. However, we have conducted pilot studies in which the connecting lines were shortened somewhat and displaced so that they no longer were directly below the overlapping dots. The results showed clearly that without connection, the effects of the context lines were reduced such that percentages of endto-end and group movement changed in the expected directions by no more than an average of $4 \%-5 \%$, with some subjects showing effects as large as $12 \%$. In addi- tion, the motion or stationarity of the lower row of stimulus dots was shown not to influence the reports of end-toend and group movement of the dots in the upper row in the present experiment. It therefore seems likely that our results cannot be entirely explained by the summation of SR and LR signals.

A second possible explanation of the present results is that there is a perceptual effect brought about by connecting the position-cue lines to the Ternus elements that is unrelated to low-level motion signals. Such an effect might be described alternatively as "unconscious inference," "taking into account," or "top-down influence," depending on one's theoretical predilection. In such a case, the final percept might plausibly be regarded as depending on the relative strengths of more or less low-level motion signals (i.e., SR and LR signals) and higher order influences. 
The present experiment was not designed to test the theoretical positions mentioned above, and it is therefore not possible to provide strong support for one or the other. Nonetheless, the results of the pilot study in which the position-cue lines were somewhat shortened and shifted do suggest that our cue effects depend on the ability of the observer to associate the cue lines with specific stimulus dots. This in turn may imply a role for an interpretive factor in the perception of our displays.

\section{REFERENCES}

ANSTIS, S. M., \& Ramachandran, V. S. (1986). Entrained path deflection in apparent motion. Vision Research, 26, 1733-1739.

Boring, E. G. (1942). Sensation and perception in the history of experimental psychology. New York: Appleton-Century.

BRADDICK, O. J. (1974). A short-range process in apparent motion. $\mathrm{Vi}$ sion Research, 13, 355-369.

BradDICK, O. J., \& ADLARD, A. J. (1978). Apparent motion and the motion detector. In J. Armington, J. Krauskopf, \& B. R. Wooten (Eds.), Visual psychophysics and physiology (pp. 417-426). New York: Academic Press.

Breitmeyer, B. G., May, J. G., \& Williams, M. C. (1988). Spatial frequency and contrast effects on percepts of bistable stroboscopic motion. Perception \& Psychophysics, 44, 525-531.

BreitMeyer, B. G., \& RitTer, A. (1986a). The role of visual pattern persistence in bistable stroboscopic motion. Vision Research, 26, 1801-1806.

BreItMeyer, B. G., \& RitTeR, A. (1986b). Visual persistence and the effect of eccentric viewing, element size, and frame duration on bistable stroboscopic motion percepts. Perception \& Psychophysics, 39, 275-280.

Chang, J. J., \& Julesz, B. (1984). Cooperative phenomena in apparent movement perception of random-dot cinematograms. Vision Research, 24, 1781-1788.

Green, M., \& ODOM, J. V. (1986). Correspondence matching in apparent motion: Evidence for three-dimensional spatial representation. Science, 233, 1427-1429.

GrossberG, S. (1989, September). A neural architecture for visual motion perception: Group and element apparent motion. Paper presented at the 12th European Conference on Visual Perception, Zichron Yaakov, Israel.

Pantle, A. J., \& Petersik, J. T. (1980). Effects of spatial parameters on the perceptual organization of a bistable motion display. Perception \& Psychophysics, 27, 307-312.

Pantle, A. J., \& Picciano, L. (1976). A multistable movement display: Evidence for two separate motion systems in human vision. Science, 193, 500-502.

Petersix, J. T. (1989). The two-process distinction in apparent mo tion. Psychological Bulletin, 106, 107-127.

PetersiK, J. T. (1990). Global cooperativity of the short-range process in apparent motion: Evidence obtained with contour-containing stimuli Perception \& Psychophysics, 47, 360-368.
Petersik, J. T., \& Pantle, A. J. (1979). Factors controlling the competing sensations produced by a bistable stroboscopic motion display. Vision Research, 19, 143-154.

ShePard, R. N., \& ZARE, S. L. (1983). Path-guided apparent motion. Science, 220, 632-634

Sigman, E., \& Rock, I. (1974). Stroboscopic movement based on perceptual intelligence. Perception, 3, 9-28.

Ternus, J. (1938). The problem of phenomenal identity. In W. D. Ellis (Ed.), A source book of Gestalt psychology (pp. 000-000). London: Routledge \& Kegan Paul.

Ullman, S. (1979). The interpretation of visual motion. Cambridge, MA: MIT Press.

\section{NOTES}

1. The characteristics of the short- and long-range processes in apparent movement are too numerous to list here. Suffice it to say that in studies using both the Ternus display (e.g., Pantle \& Picciano, 1976) and random-dot cinematograms (e.g., Braddick, 1974), the long-range process has been shown to be favored by long spatial displacements and ISIs relative to the short-range process. Furthermore, dichoptic viewing, illuminated interstimulus intervals, and low stimulus contrasts are relatively unfavorable to the short-range process. For a further review of the characteristics of these hypothetical processes, as well as a discussion of their relationship to the Ternus display, see Petersik (1989).

2. Recent work by Breitmeyer and others (e.g., Breitmeyer, May, \& Williams, 1988; Breitmeyer \& Ritter, 1986a, 1986b) has shown a relationship between conditions that favor end-to-end movement and those that favor pattern persistence. This in turn has led some individuals to the conclusion that pattern persistence "explains" end-to-end movement and that it is not necessary to implicate the (or any) SR process. We find this to be a rather narrow extrapolation of Breitmeyer's findings. First, the similarities between the conditions that favor the SR process in random-dot cinematograms and those that favor end-to-end movement in the Ternus display are far too many to be ignored, and as of now, pattern persistence has not been conclusively shown to be related to SR movement in random-dot displays. Second, Breitmeyer and Ritter (1986a) have themselves acknowledged that pattern persistence alone cannot account for all aspects of movement in the Ternus display: "although pattern persistence may contribute to motion perception in bistable motion displays, the temporal parameters of static pattern persistence do not of themselves explain what may be substantially different and, at times, overriding dynamic properties of motion detection mechanisms" (p. 1806). Third, Grossberg (1989) has recently presented a neural-network model that successfully integrates SR and LR processes and pattern persistence. Along with Breitmeyer and Grossberg, we favor attempts to synthesize explanations of phenomena that may, at first glance, appear to be unrelated, rather than attempts to establish simple, single-factor explanations.

(Manuscript received January 4, 1990; revision accepted for publication March 27, 1990.) 